\title{
Efficacy of long-term anticoagulant treatment in subgroups of patients after myocardial infarction
}

\author{
Paul F M M van Bergen, Jaap W Deckers, Jan J C Jonker, Ron T van Domburg,
} Aida J Azar, Albert Hofman

\author{
ASPECT \\ Coordinating Centre, \\ Rotterdam, The \\ Netherlands \\ P F M M van Bergen \\ J J C Jonker \\ A J Azar \\ Thoraxcentre, \\ University Hospital \\ Rotterdam, The \\ Netherlands \\ J W Deckers \\ R T van Domburg \\ Erasmus University \\ Medical School, \\ Department of \\ Epidemiology and \\ Biostatistics, \\ Rotterdam, The \\ Netherlands \\ P F M M van Bergen \\ J J C Jonker \\ A Hofman \\ Correspondence to: \\ Dr P F M M van Bergen, \\ Rotterdam Medical \\ Research Foundation, \\ (ROMERES), \\ Mathenesserlaan 247, 3021 \\ HC, Rotterdam, The \\ Netherlands. \\ Accepted for publication \\ 21 December 1994
}

\begin{abstract}
Objective-To investigate the efficacy of long term oral anticoagulant treatment in subgroups of patients after myocardial infarction.

Design-Analysis of the effect of anticoagulant treatment in subgroups of hospital survivors of myocardial infarction based upon age, gender, history of hypertension, previous myocardial infarction, smoking habits, diabetes mellitus, Killip class, anterior location of infarction, thrombolytic therapy, and use of $\beta$ blockers.
\end{abstract}

Subjects-Participants of a multicentre, randomised, double blind, placebo controlled trial that assessed the effect of oral anticoagulant treatment on mortality as well as cerebrovascular and cardiovascular morbidity in 3404 hospital survivors of acute myocardial infarction.

Main outcome measures-The effect of anticoagulant treatment on recurrent myocardial infarction, cerebrovascular events, and vascular events (the composite endpoint of reinfarction, cerebrovascular event, and vascular death).

Results-Long term anticoagulant treatment was associated with a reduction in mortality of $10 \%$ (95\% confidence interval $-11 \%$ to $27 \%$ ), recurrent myocardial infarction of $53 \%(41 \%$ to $62 \%)$, cerebrovascular events of $40 \%(10 \%$ to $60 \%)$ and vascular events of $35 \%$ (24\% to $45 \%)$. Treatment effect with respect to recurrent myocardial infarction was comparable among all subgroups of patients. Although treatment effect appeared to be somewhat smaller in females than in males $(-11 \% v-45 \%)$, and in patients with diabetes compared to those without $(-14 \% v-42 \%)$ with respect to vascular events, none of these differences reached statistical significance. In multivariate analysis, more advanced age, previous myocardial infarction, diabetes mellitus, and heart failure during admission were independently associated with increased incidence of cardiovascular complications.

Conclusions-The relative benefit of long term anticoagulant therapy in survivors of myocardial infarction is not modified by known prognostic factors for cardiovascular disease.

\section{(Br Heart f 1995;74:117-121)}

Keywords: effect modification; anticoagulant treatment; myo-cardial infarction; subgroup analysis; prognosis
Patients who survive an acute myocardial infarct are at increased risk for new arterial thromboembolism. Recent clinical trials have shown that long term oral anticoagulant treatment with a target international normalised ratio (INR) of $2 \cdot 8-4 \cdot 8$ reduces the incidence of such complications. ${ }^{12}$ However, oral anticoagulant treatment has also been associated with an increased risk of bleeding complications which can sometimes be fatal or cause severe disability as in the case of intracranial haemorrhage..$^{3-5}$ Determination of clinical variables that identify subsets of patients who do not benefit from anticoagulant treatment could preclude them from unnecessary exposure to the potentially adverse effects of treatment. Results of a recent subgroup analysis of the Warfarin Re-infarction Study indicated that the presence of diabetes mellitus and previous myocardial infarction could offset the beneficial effect of long term warfarin treatment. In addition, it was also suggested that older patients had a relatively smaller treatment benefit. ${ }^{6}$ Although results of subgroup analysis should always be viewed with caution, since the large number of comparisons in such an analysis increases the possibility of chance findings, confirmation of such associations in another trial would of course substantiate these assumptions. We therefore performed a subgroup analysis of the ASPECT (Anticoagulants in the Secondary Prevention of Events in Coronary Thrombosis) trial, which investigated the effect of long term anticoagulant treatment relative to placebo in 3404 hospital survivors of myocardial infarction.

\section{Methods}

PATIENTS

Details of the ASPECT trial, a multicentre, randomised, double blind, placebo controlled trial that assessed the efficacy of long term anticoagulant therapy after myocardial infarction, have been published previously. ${ }^{2}$ In short, the ASPECT trial comprised 3404 hospital survivors of an acute myocardial infarct who were randomised between September 1986 and January 1992 to oral anticoagulant therapy with the coumarin derivatives phenprocoumon or acenocoumarol $(n=1700)$, or matching placebo $(n=1704)$, within on average four days following hospital discharge. Patients with an indication for oral anticoagulant therapy (for example, chronic atrial fibrillation, left ventricular thrombus or aneurysm, or cardiomyopathy), or who were on anticoagulant treatment within a period of six 
months preceding the qualifying myocardial infarction, with increased bleeding tendency, anticipated revascularisation procedure, malignant disease with poor prognosis, mental disorder, pregnancy, or other household members receiving oral anticoagulant therapy, were not eligible. Oral anticoagulant therapy was adjusted individually, with a prothrombin target range of 2:8-4.8 INR. Treatment allocation was double blind. Trial medication was continued until the end of the trial follow up on June 30, 1992. The median duration of follow up was 37 months. The study was approved by the ethics committees of the participating hospitals and all patients had given oral informed consent.

\section{EVENT DEFINITION AND TRIAL OUTCOME}

All events were classified blindly by a mortality and morbidity classification committee. We considered the following endpoints for this subgroup analysis: recurrent myocardial infarction, cerebrovascular event, and vascular event. Recurrent myocardial infarction was defined as a history of chest discomfort with a duration exceeding 30 minutes and serial enzyme pattern typical for myocardial infarction, with at least one cardiac enzyme exceeding twice the upper reference limit, or the development of new $Q$ waves ( $>0.03 \mathrm{~s}$ ) on the standard 12-lead electrocardiogram. Patients who survived the onset of symptoms of an acute myocardial infarction for at least one hour and died subsequently were also classified as having suffered a recurrent myocardial infarction. Cerebrovascular event included haemorrhagic and ischaemic stroke and transient ischaemic attack. Vascular event was defined as the composite endpoint of either vascular death, recurrent myocardial infarction, or cerebrovascular event. The primary endpoint of the main trial was (total) mortality. Because anticoagulant treatment was not associated with a significant reduction in mortality-there were 170 deaths in anticoagu-

Table 1 Baseline characteristics of randomised patients according to therapy group

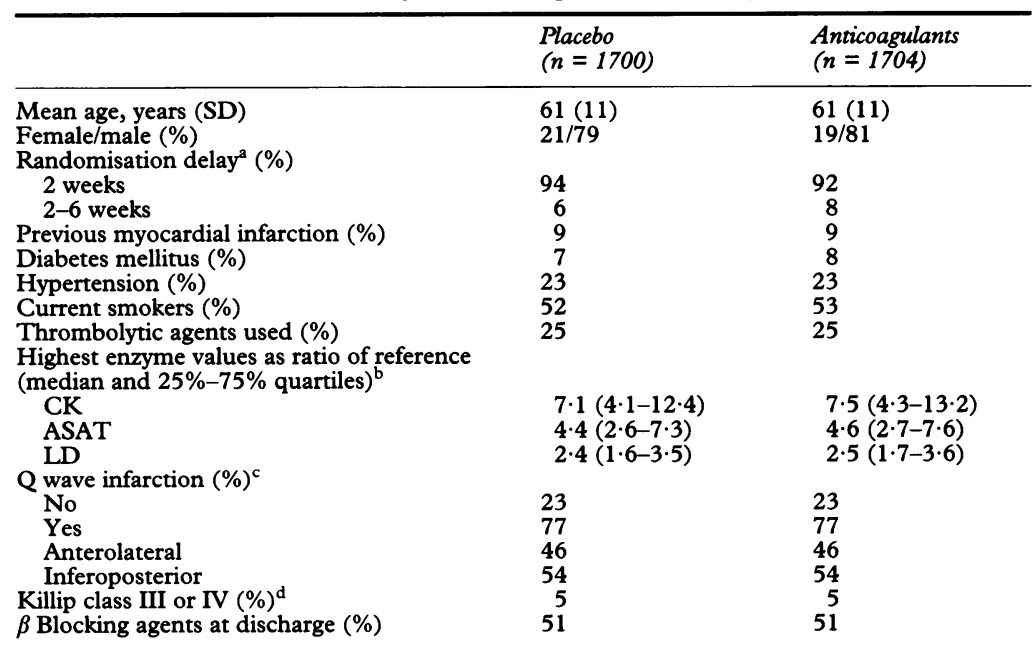

AC, anticoagulated; $\mathrm{CK}$, creatine kinase; ASAT, serum aspartate aminotransferase; LD, lactate dehydrogenase.

a Time from hospital discharge to randomisation.

${ }^{b}$ Expressed as multiple of the upper limit of normal.

${ }^{c} Q$ waves ${ }^{3} 0.03 \mathrm{~s}$ and ${ }^{3} 0.1 \mathrm{mV}$.

d Highest Killip or MIRU class reached in hospital. lated and 189 in placebo patients, a reduction of $10 \%$ ( $95 \%$ confidence interval $-11 \%$ to $27 \%$ - this endpoint was not considered in the subgroup analysis.

Recurrent myocardial infarction was observed in 114 anticoagulated patients versus 242 placebo patients, a highly significant reduction of $53 \%$ ( $41 \%$ to $62 \%)$, cerebrovascular events in 37 anticoagulated versus 62 placebo patients, a reduction of $40 \%(10 \%$ to $60 \%$ ), and any vascular event in 239 anticoagulated versus 366 placebo patients, a reduction of $35 \%(24 \%$ to $45 \%)$. Major bleeding complications were seen in 73 anticoagulated and 19 placebo patients (relative risk 3.9, $95 \%$ confidence interval $2 \cdot 3$ to $6 \cdot 4$ ).

\section{DATA ANALYSIS}

Risk (hazard) estimates and their corresponding $95 \%$ confidence intervals (CI) associated with anticoagulant treatment relative to placebo were calculated with the Cox model in clinically defined subgroups of patients with the described endpoints as the dependent variable. In addition, clinical variables known to affect prognosis were inserted into a multivariate Cox proportional hazards model to assess their independent contribution to outcome. Effect modification of anticoagulant therapy was explored by the introduction of interaction terms in the model. Other group comparisons were made with Student's $t$ test and the $\chi^{2}$ test. All analyses were performed according to the "intention to treat" principle. All reported $P$ values are two tailed, and $P$ values $<0.05$ were considered statistically significant.

\section{Results}

\section{PATIENTS}

Baseline characteristics of the patients are presented in table 1 . No important differences were observed between the treatment groups for any of the baseline variables. Approximately $20 \%$ of the patients were women, less than $8 \%$ had diabetes, $9 \%$ had suffered a previous myocardial infarction before enrolment, and $25 \%$ had been treated with thrombolytic agents.

\section{TREATMENT EFFECT IN SUBGROUPS}

The relative effects of anticoagulant treatment on recurrent myocardial infarction and vascular event within subgroups of clinically defined patients are displayed in figs 1 and 2 . Effect estimates on the outcome cerebrovascular event, while highly significant in the overall analysis, showed large variations and wide corresponding confidence intervals in almost all subgroups, because of the relatively small number of cerebrovascular accidents in specific categories. As a consequence, this endpoint was not considered further in subgroup analyses.

The effect of anticoagulant treatment on reinfarction over the strata was fairly homogeneous, with only minor and non-significant attenuation of the treatment effect in women and in patients with previous myocardial 
Recurrent myocardial infarction

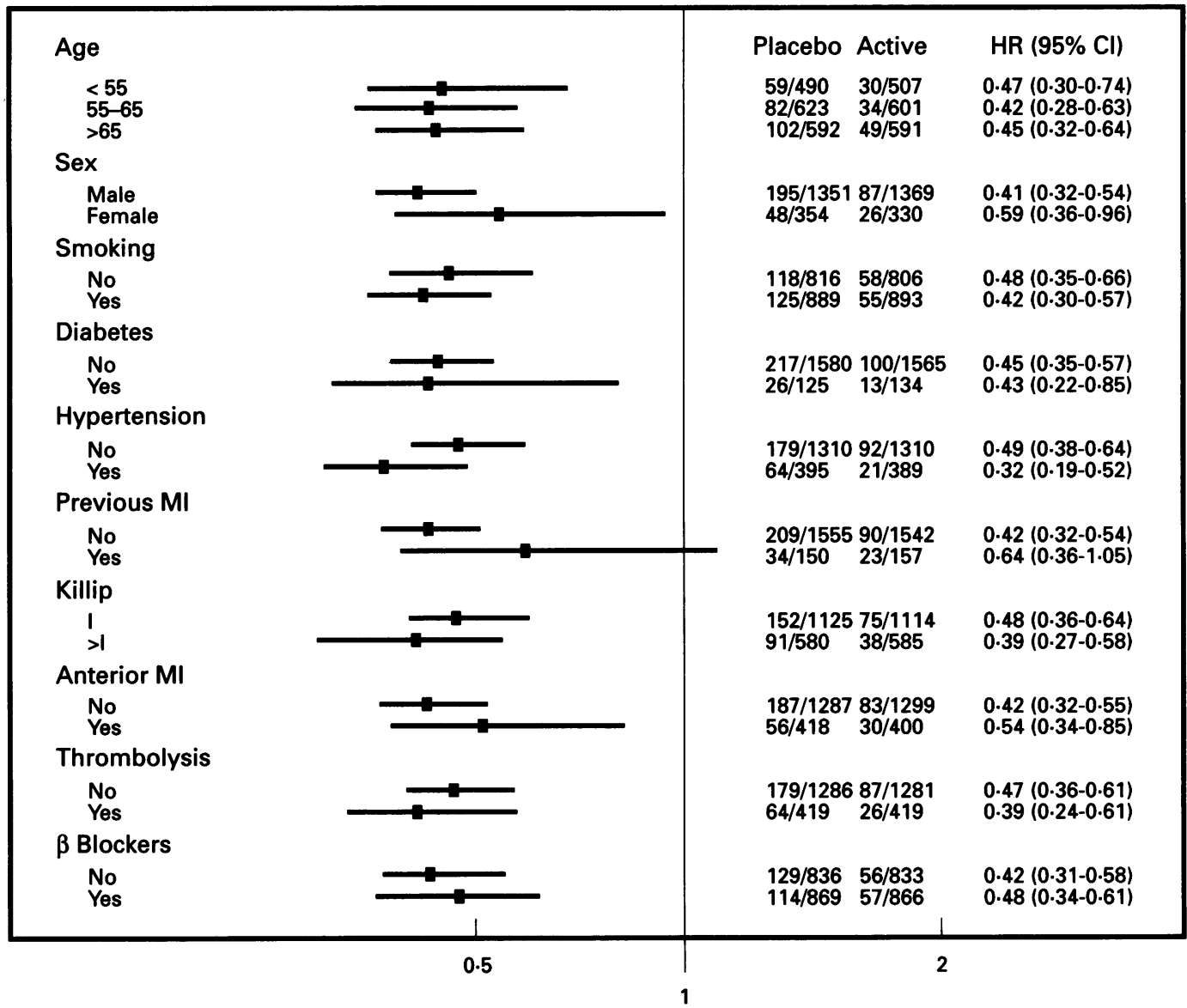

Figure 1 Effect of oral anticoagulant treatment with respect to recurrent myocardial infarction in clinically defined subgroups of patients. Vertical bars indicate best effect estimate and horizontal bars confidence interval. MI, myocardial infarction.

Vascular event

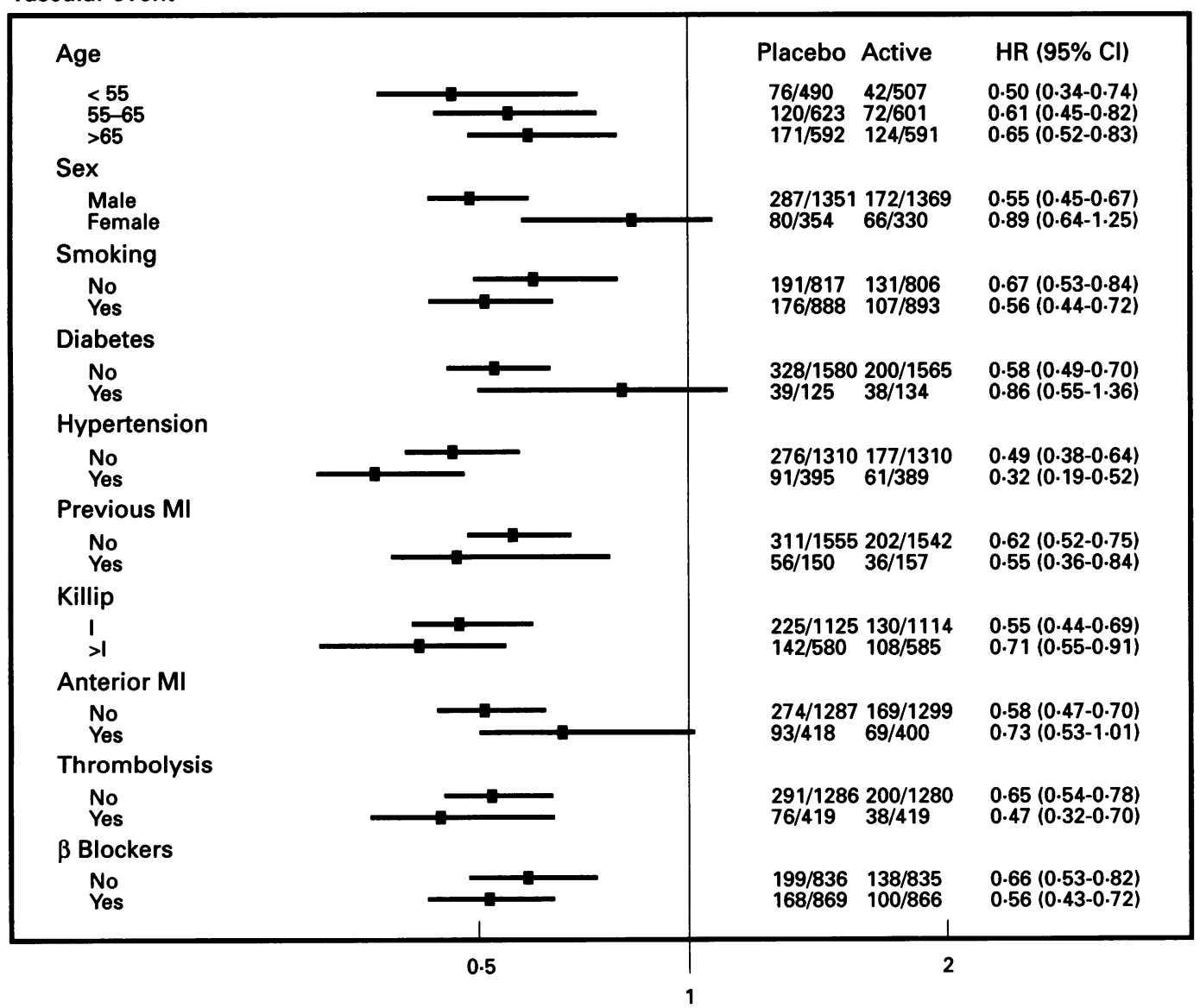

Figure 2 Effect of oral anticoagulant treatment with respect to vascular event in clinically defined subgroups of patients. MI, myocardial infarction. 
Table 2 Adjusted relative risk estimates (and 95\% confidence intervals) of prognostic factors in post myocardial infarction (MI) patients *

\begin{tabular}{lllll}
\hline & \multicolumn{4}{l}{ Relative risk (95\% confidence interval) } \\
\cline { 2 - 5 } & Recurrent MI & Cerebrovascular event & Vascular event & Mortality \\
\hline Anticoagulant therapy & $0.44(0.35,0.55)$ & $0.61(0.40,0.92)$ & $0.61(0.52,0.72)$ & $0.90(0.73,1 \cdot 11)$ \\
Age (increment of 1 year) & $1.02(1.01,1.03)$ & $1.05(1.03,1.08)$ & $1.03(1.02,1.04)$ & $1.07(1.05,1.08)$ \\
Previous MI & $2.00(1.47,2.64)$ & $1.08(0.57,2.07)$ & $1.79(1.43,2 \cdot 26)$ & $1.67(1.25,2 \cdot 23)$ \\
Diabetes & $1.45(1.03,2.05)$ & $2.32(1.39,3.90)$ & $1.59(1.24,2.04)$ & $1.34(0.96,1.85)$ \\
Thrombolysis & $1.42(1.09,1.85)$ & $0.69(0.36,1.32)$ & $1.01(0.82,1.25)$ & $0.73(0.53,1.02)$ \\
Killip class $>1$ & $1.02(0.81,1.28)$ & $0.88(0.58,1.36)$ & $1.22(1.03,1.45)$ & $1.67(1.35,2.08)$ \\
$\beta$ Blockers & $0.97(0.78,1.21)$ & $1.29(0.85,1.95)$ & $0.86(0.73,1.07)$ & $0.70(0.56,0.87)$ \\
\hline
\end{tabular}

${ }^{\star}$ Gender, smoking, hypertension, and anterior infarction were not statistically significantly associated with any of the outcome events.

infarction. For instance, the risk (hazard) of recurrent myocardial infarction associated with anticoagulant therapy in women was 0.59 (95\% confidence intervals $0.36,0.96$ ), indicating a risk reduction of $41 \%$ relative to a $59 \%$ decrease in men. Risk reductions associated with anticoagulant in patients with and without previous myocardial infarction were $58 \%$ and $36 \%$, with overlapping confidence limits. The benefit of treatment was almost identical in patients with and without diabetes.

Obviously, the composite diagnosis "vascular event" comprised the highest number of endpoints. The absolute risk of cardiovascular complications was very high in patients with diabetes: the event rate was $31 \%$ in diabetics, compared to $17 \%$ in non-diabetics. A gradual decrease in treatment effectiveness was observed with increasing age, without statistically significant differences among the age groups. The risk reduction in women was $11 \%(95 \% \mathrm{CI}-25 \%, 36 \%)$ and $14 \%(95 \%$ CI $-36 \%, 45 \%)$ in patients with diabetes, compared to reductions of $45 \%$ in men and $42 \%$ in non-diabetic patients, respectively. These differences did not reach statistical significance.

\section{ADDITIONAL ANALYSES}

Results of multivariate analyses, including for completeness the endpoint mortality, are presented in table 2. Adjustment for other factors that affected prognosis did not appreciably change the risk reductions associated with treatment. After adjustment, anticoagulant treatment was associated with a $56 \%$ lower incidence of recurrent myocardial infarction (relative risk $0.44,95 \%$ confidence interval $0.35,0.55)$. More advanced age, previous myocardial infarction, the presence of diabetes, and the use of thrombolytic therapy were all associated with a higher incidence of recurrent myocardial infarction during follow up. The use of thrombolytics was strongly protective against mortality, however, and neutral with respect to the combined endpoint "vascular event". In multivariate analysis, female gender was not independently associated with increased risk of recurrent myocardial infarction or vascular event. Anticoagulant treatment was associated with an adjusted $39 \%$ lower incidence of cerebrovascular events, with age and diabetes being important independent predictors. Again, the strongest predictors of death were more advanced age, diabetes, Killip class $>1$, and previous myocardial infarction, while $\beta$ blockers and thrombolytic treatment were associated with lower all cause mortality.

The introduction of an interaction term between anticoagulant treatment and gender did not influence the effect of treatment with respect to the occurrence of vascular events. Additional review of the data revealed that, on average, women were older than men ( $65 v 59$ years), smoked less ( $46 \% v 67 \%)$, and more often had diabetes $(14 \% v 6 \%$, all $\mathrm{P}<0.001)$, characteristics that appeared to be associated with a smaller benefit from anticoagulant therapy (fig 2). Interaction terms were also introduced to elucidate the relationship of anticoagulant treatment, diabetes, and vascular events, but no significant associations could be established.

\section{Discussion}

The results of the present analysis show that the relative effect of long term oral anticoagulant treatment on various thromboembolic endpoints after myocardial infarction is not modified among clinically defined subgroups of patients. The results of our analysis therefore do not confirm that anticoagulant treatment may be less effective in patients with previous myocardial infarction or diabetes, as has been suggested previously.

In spite of their potentially important clinical implications, subgroup analyses of anticoagulant treatment after myocardial infarction are scarce, probably because only a few trials were of sufficient size to permit such an analysis. The International Anticoagulant Review Group addressed this problem by pooling data of nine controlled studies. ${ }^{7}$ As in the current analysis, that study also reported a modest treatment effect in women. This finding may be related to the more advanced age of women relative to the men, as in our study.

There was no interaction of previous myocardial infarction with the effect of anticoagulant treatment on recurrent myocardial infarction. These findings are in accordance with the results of the Veterans Administration Study, ${ }^{8}$ but, again, are at variance with the outcome of the subgroup analysis of the Warfarin Re-infarction Study. Moreover, in that study recurrence of myocardial infarction was not reduced by anticoagulant treatment in the presence of diabetes. ${ }^{6}$ As discussed earlier, our findings may be consis- 
tent with a somewhat attenuated effect of anticoagulant treatment with respect to vascular events in patients with diabetes mellitus, but no modification was shown with respect to the endpoint "recurrent myocardial infarction". The higher overall rate of cardiovascular complications in the WARIS trial compared to the ASPECT trial may be important with regard to these discrepant outcomes.

Some limitations of subgroup analysis should be emphasised. Foremost and as a matter of course, statistical power in subgroups is negatively affected by the small number of patients or endpoints available for analysis, as in this case with respect to stroke. Also, when several subgroups are investigated without a previous hypothesis, the chance of random associations increases. It may therefore be appropriate to adjust the level of significance to a more stringent degree to compensate for multiple testing. In the present subgroup analyses, however, despite the large number of comparisons that was made, statistical significance was not even approached at the level of $5 \%$, and adjustment for multiplicity was therefore inconsequential and unnecessary.

Multivariate analysis was performed to identify variables that showed an independent association with the selected endpoints. This analysis confirmed the association of increasing age, previous myocardial infarction, diabetes, and clinical signs of heart failure during admission with cardiovascular morbidity and mortality after myocardial infarction, ${ }^{9-11}$ and the beneficial effect of the use of $\beta$ blockers. ${ }^{12}{ }^{13}$ The lack of association between anterior infarction and mortality probably resulted from selection of low risk patients in this trial. The effect of thrombolysis on mortality corresponds with earlier findings of the ISIS-2 study. ${ }^{14-16}$

In view of the fact that none of the considered variables showed a significant modification of treatment effect, it may be argued that all patients have equal relative benefit from anticoagulant treatment and should thus be treated. Although this may be a valid statement, multivariate analysis revealed a number of distinct features of patients at increased risk for subsequent cardiovascular events. In this respect it is of particular importance to note that thrombolysis confers a profound reduc- tion in mortality but may otherwise increase the risk of recurrent myocardial infarction.

In summary, the results of this analysis indicate that the effects of anticoagulant treatment after myocardial infarction are not modified in clinically defined subgroups of patients and that anticoagulant treatment may in particular be considered after myocardial infarction in patients with characteristics associated with increased risk of cardiovascular complications.

Supported by a main grant from the Praeventiefonds, The Netherlands (28-978) and a subsidiary grant from the Netherlands Thrombosis Foundation, The Netherlands.

1 Smith $\mathrm{P}$, Arnesen $\mathrm{H}$, Holme I. The effect of warfarin on mortality and reinfarction after myocardial infarction. $N$ Engl f Med 1990;323:147-52.

2 The ASPECT research group. Effect of long-term oral anticoagulant treatment on mortality and cardiovascular morbidity after myocardial infarction. Lancet 1994;343 499-503

3 Levine MN, Raskob G, Hirsch J. Haemorrhagic complications of long-term anticoagulant therapy. Chest 1989 95(2) suppl:26-36S.

4 Fogelholm R, Eskola K, Kiminkinen T, Kunnamo I. Anticoagulant treatment as a risk factor for primary intracerebral haemorrhage. $\mathcal{F}$ Neurol Neurosurg Psychiatry
$1992 ; 55: 1121-4$.

5 Wintzen AR, Jonge $\mathrm{H}$ de, Loeliger EA, Bots GTAM. The risk of intracerebral haemorrhage during oral anticoagulant treatment: a population study. Ann Neurol 1984;16 553-8.

6 Smith P, Arnesen H, Abdelnoor M. Effects of long-term anticoagulant therapy in subgroups after acute myocardial infarction. Arch Intern Med 1992;152:993-7.

7 International Anticoagulant Review Group. Collaborative analysis of long-term anticoagulant administration after acute myocardial infarction. Lancet 1970;i:203-9.

8 Ebert RV, Borden CW, Hipp HR, Holzman D, Lyon AF, Schnaper $H$. Long-term anticoagulant therapy after myocardial infarction. $\mathscr{f} A M A$ 1969;207:2263-7.

9 Norris RM, Caughey DE, Mercer CJ, Scott PJ. Prognosis after myocardial infarction. Six year follow-up. Br Heart $\mathcal{f}$ 1974;36:786-90.

10 Merrilees M, Scott PJ, Norris RM. Prognosis after myocardial infarction: results of 15 year follow up. $B M 9$ 1984;288:356-9.

11 Martin CA, Thompson PL, Armstrong BK, Hobbs MST, de Klerk N. Long-term prognosis after recovery from myocardial infarction: a nine year follow-up of the Perth Coronary Register. Circulation 1983;68:961-9.

12 The Norwegian Multicenter Study Group. Timololinduced reduction in mortality and reinfarction in patients surviving acute myocardial infarction. $N$ Engl $\mathcal{F}$ patients surviving acute

13 Beta-blocker Heart Attack Study Research Group. A randomised trial of propanolol in patients with acute myocardial infarction. $\mathscr{F} A M A$ 1982;247:1707-14.

14 Stone PH, Raabe DS, Jaffe AS. Prognostic significance of location and type of myocardial infarction: independent adverse outcome associated with anterior location. $7 \mathrm{Am}$ Coll Cardiol 1988;11:453-63.

15 Geltman EM, Ehsani AA, Campbell MK, Schechtman K, Roberts R, Sobel BE. The influence of location and extent of myocardial infarction on long-term ventricular dysrhythmia and mortality. Circulation 1979;60:805-14.

dysrhythmia and mortality. Circulation $1979 ; 60: 805-14$.
16 ISIS-2 Collaborative Group. A randomised trial of intravenous streptokinase, oral aspirin, both, or neither among 17187 cases of suspected acute myocardial infarction. Lancet 1988;ii:349-60. 